\title{
Building Blocks of Zeolites on an Aluminosilicate Ultra-Thin Film.
}

\author{
J. Anibal Boscoboinik*, Xin Yu, Bing Yang, Shamil Shaikhutdinov and Hans-Joachim Freund. \\ Fritz-Haber-Institut der Max-Planck-Gesellschaft, Faradayweg 4 - 6, 14195 Berlin, Germany. \\ KEYWORDS: Zeolites, Aluminosilicates, Surface Science, Building Units, Topochemistry.
}

Supporting Information Placeholder

\begin{abstract}
Some of the basic building units found on many zeolites, as well as larger fragments found in specific cases, were imaged within the structure of an aluminosilicate ultra-thin film synthesized on a $\mathrm{Ru}(0001)$ substrate. These units have been suggested as possible precursors for the wide variety of zeolites reported to date. The reported structure consists of a planar sheet of double $\mathrm{N}$-membered rings (polygonal prisms) sharing four-membered ring faces in the planes normal to the surface. The units are mostly double six-membered rings $(d 6 r)$, with a significant population of pairs of double four- and eightmembered rings $(d 4 r$ and $d 8 r$ ) and pairs of double five- and seven-membered rings ( $d 5 r$ and $d 7 r$ ), among other minority species. In particular, there is significant increase of the $d 4 r$ and $d 8 r$ unit in this aluminum-containing film, when compared to pure-silica ones prepared in the same manner. These building blocks appear $25(d 6 r), 17(d 4 r)$ and $5(d 8 r)$ times, in the set of 176 unique zeolite frameworks reported up to 2007. In addition, larger ensembles of rings observed in this system can be related to more complex ensembles in zeolites, such the $\alpha$-cage in zeolite $\mathrm{A}$. The structures were here identified by a combination of scanning tunneling microscopy, low energy electron diffraction, infrared reflection absorption spectroscopy and x-ray photoelectron spectroscopy experiments.
\end{abstract}

The structure of an aluminosilicate film composed of a single layer of polygonal prisms, determined with atomic resolution, is analyzed in this work, revealing the presence of some of the most frequently found building blocks of zeolites. These include units that have been repeatedly proposed as precursors for the formation of these crystalline porous materials. [Cundy2005] The structure of zeolites and zeolite-related architectures is of great importance as it is intrinsically related to the many applications of these materials. In terms of the chemistry of these systems, it has been shown that small variations in structural parameters near the active sites can have a significant effect on their catalytic activity. [Eichler97, Brändle98, Katada2009] In addition to this, there is substantial interest from a fundamental point of view, as they provide a fascinating subject of study for the field of topology. This is reflected in the millions of hypothetical zeolites structures that have been proposed just on topological basis, [Foster] in addition to the 194 structures existent as of November 2010 ( 40 found in nature and 154 synthet- ic). [Baerlocher] Additionally, resolving the structure at the atomic level can lead to a better understating of the mechanism by which the frameworks are assembled, which is currently a topic under active debate. [Cundy2005, Cejka2010] We recently reported the successful preparation a two-dimensional model system for zeolites which can be studied by scanning probe techniques to that level of detail and under very controlled conditions in an ultra-high vacuum environment. [Bosco2012]

Zeolites are crystalline materials composed of a network of tetrahedra, where the center of the tetrahedron is an atom 'T' (Si or $\mathrm{Al}$ ) which is linked to four other $\mathrm{T}$-atoms by an oxygen bridge. The tetrahedron $\mathrm{TO}_{4 / 2}$ is known as the primary building unit. These can arrange into larger structures called secondary building units (SBU's), which are defined such that the whole framework can be made of only one type of unit repeating in space. Some of the 23 SBU's known to date that have been found in at least two zeolite structures are shown in figure 1 . They are shown by connected dots where the dots represent the tetrahedral atoms, while the oxygen atoms are excluded in this representation. For the complete list see ref. [Atlas2007].

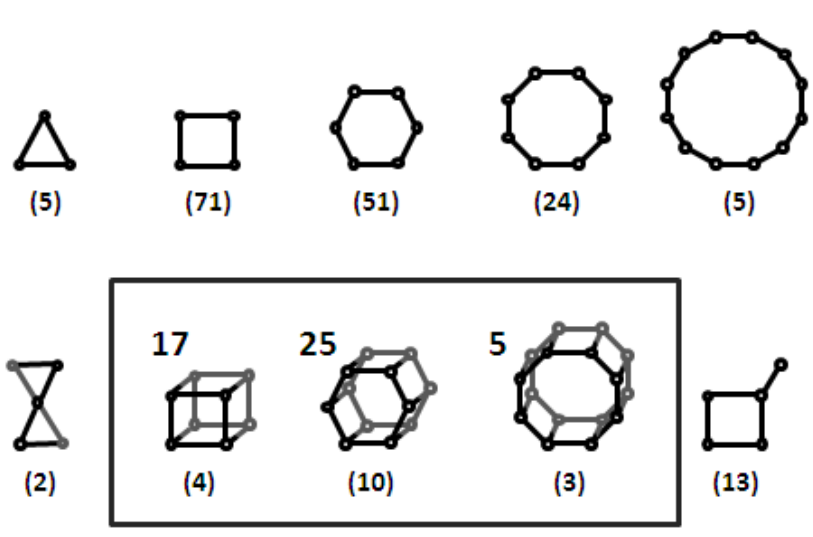

Figure 1. Connected-dots representation of some of the secondary building units of zeolites (Figure adapted from the "Atlas of Zeolite Framework Types"). [Atlas2007]

The number under each SBU represents the notation used for that unit and the number in parenthesis represents the instances of occurrence. [Atlas2007] The simplest SBU's are rings (polygons) of different sizes, i.e.: 3-, 4-, 6-, 8- and 12-membered rings (MR's). An excellent review on the topology of zeolites 
and related materials was given by Smith. [Smith88] Another definition to account for the building blocks of zeolites, which leaves out the restriction of uniqueness of such motifs in the framework, is that of the composite building units (CBU's). This includes therefore a much more extensive list of units and it is useful for making comparisons between zeolites that share some of these building blocks. Some of the most extensively occurring CBU's are the double 4-, 6- and 8-membered rings, named $d 4 r, d 6 r$ and $d 8 r$ respectively, according to the CBU's notation. [Atlas2007] These prisms are also SBU's, and they are emphasized in figure 1. The number of zeolites in which these three units have been found is written in bold face in the upperleft corner of the units in the figure. Frameworks can also be build from chains, such as the double zigzag chain and the double crankshaft chain, both of which are made of 4 membered rings. [Atlas2007]

A close relation exists between layered aluminosilicates and three-dimensional zeolite structures. A clear example of this is the transformation of $\mathrm{Ba}^{2+}$ substituted zeolite $\mathrm{A}$ (a.k.a. LTA) into the layered barium aluminosilicate known as hexacelsian upon thermal treatment. [Djordjevic2001] This layered aluminosilicate was first described by Yoshiki in 1951, [Yoshiki51] and each layer has the same connectivity of the silica film described by Loffler et al. [Loffler2010] but with half of the Si atoms substituted by $\mathrm{Al}$ and sheets of $\mathrm{Ba}^{2+}$ separating them and stabilizing the framework charge. Moreover, we were recently able to synthesize an Al-substituted system of comparable characteristics and showed that it can be successfully used as a model system for zeolites, from the chemistry point of view, by generating highly acidic bridging hydroxyls at high $\mathrm{Al}$ contents. [Bosco2012] The structure of this system shows striking similarities with different zeolites and shows features that are absent in analogous pure-silica cases. Due to the high importance of zeolites, the structural features of the aforementioned system deserve a detailed description and analysis and this is explored in the present work.

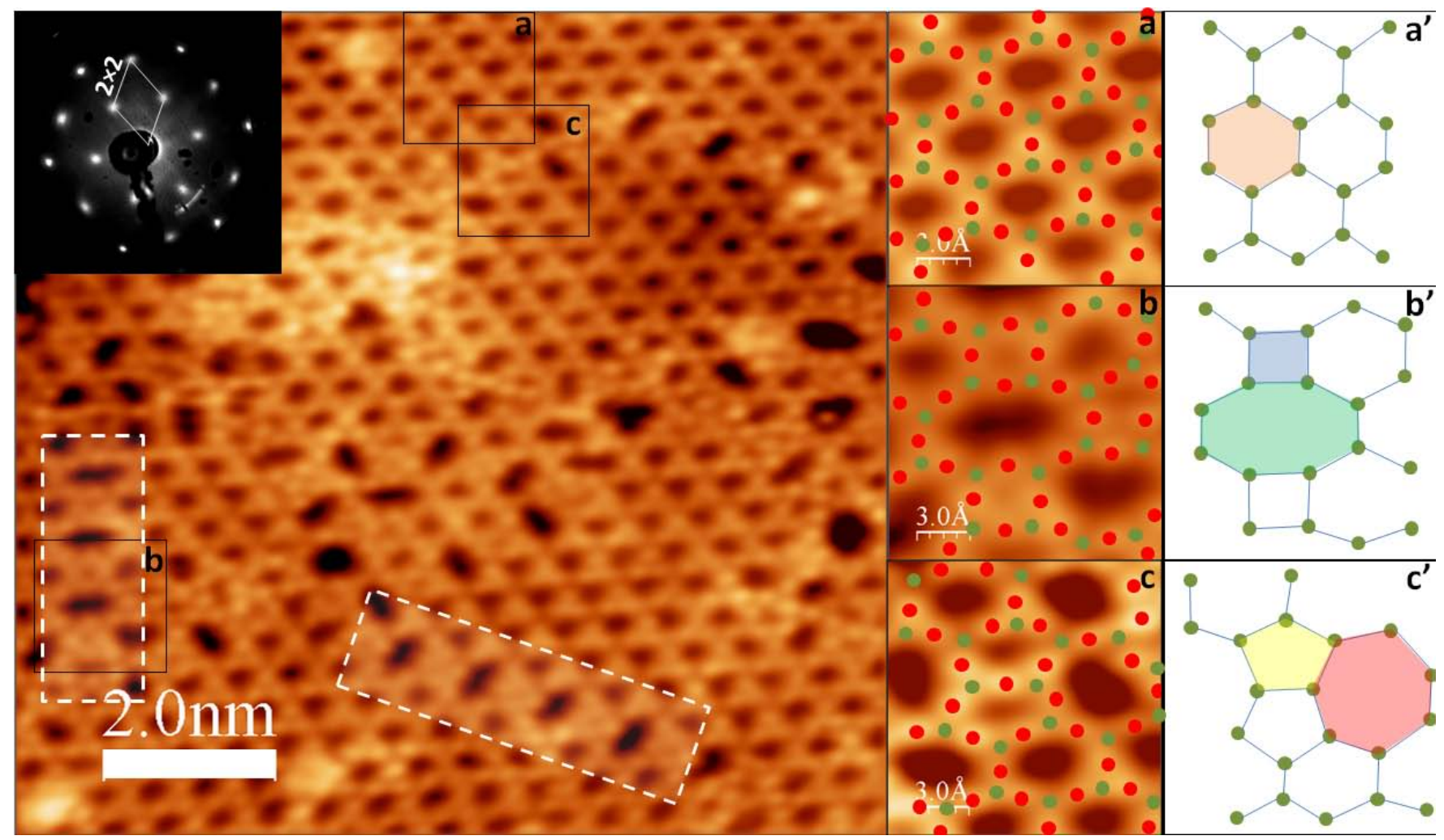

Figure 2. Left, STM image showing the morphology of the surface. The bright spots correspond to the position of the O atoms. The tetrahedral atoms $\mathrm{T}$ are located in the middle of each group of three $\mathrm{O}$ atoms, and the forth $\mathrm{O}$ atom, to which the $\mathrm{T}$ atom is bonded, is located beneath the $\mathrm{T}$ atom, connecting to the bottom layer. Insets $\mathbf{a}, \mathbf{b}$ and $\mathbf{c}$ correspond to regions containing $d 6 r, d 4 r+d 8 r$ and $d 5 r+d 7 r$ respectively. Red dots show the location of the $\mathrm{O}$ atoms while the green dots show where the $\mathrm{T}$ atoms are. Insets a', b' and c' show a connected-dots representation, where the dots correspond to the location of the $\mathrm{T}$ atoms.

The preparation and the experiments reported here were performed in an ultrahigh-vacuum system counting with the following techniques: low energy electron diffraction (LEED), x-ray photoelectron spectroscopy (XPS), infrared reflection absorption spectroscopy (IRAS), and scanning tunneling microscopy
(STM). The film was prepared by subsequently depositing $0.64 \mathrm{ML}\left(1 \mathrm{ML}=1.57 \times 10^{15}\right.$ atoms $\left./ \mathrm{cm}^{2}\right)$ of $\mathrm{Si}$ and $\sim 0.36 \mathrm{ML}$ of $\mathrm{Al}$ on a $3 \mathrm{O}-(2 \times 2) / \mathrm{Ru}(0001)$ surface under an $\mathrm{O}_{2}$ pressure of $2 \times 10^{-7}$ mbar. The film was then oxidized by exposing it to a pressure of $\mathrm{O}_{2}$ of $3 \times 10^{-6}$ mbar while heating the sample up to 
$\sim 950{ }^{\circ} \mathrm{C}$, keeping it at this temperature for 10 minutes and then slowly cooling it down always under $\mathrm{O}_{2}$ ambient. More detailed experimental details are given in the supporting information section.

The aluminum and silicon contents were determined by XPS of the Al2s and Si2p core levels. A correction factor of 1.17 was applied to the ratio of the peak areas $\mathrm{Als} 2 / \mathrm{Si} 2 \mathrm{p}$ to account for the different sensitivity factors of these two levels, based on experimental data obtained for an electron analyzer similar to the one used in our experiments. [Wagner81] This yielded a molar ratio $\mathrm{Al} / \mathrm{Si}=0.56$. (XPS spectra shown in the supporting information). The different motifs found in this structure can be clearly identified with high-resolution by STM. Figure 2 shows an STM image containing a variety of distinct features. Similar to the case of an ordered pure-silica bilayer system reported previously, [Loffler2010] the majority of the framework is composed of double 6-membered rings (d6r). Although only the immediate surface can be seen in the image, evidence of the same structure repeating symmetrically below is given by IRAS. [Bosco2012] The infrared spectrum for this system, showing sharp phonon vibration peaks at $1277 \mathrm{~cm}^{-1}$ and $702 \mathrm{~cm}^{-1}$ is shown in the supporting information. The inset at the upperleft corner of figure 2 shows the LEED pattern of this film, taken at a beam energy of $60 \mathrm{eV}$, where spots corresponding to a $(2 \times 2)$ structure, with respect to the $\mathrm{Ru}(0001)$ underlying lattice, are observed. In addition to the $d 6 r$, direct space imaging by STM (Figure 2) shows the presence of other structures lacking long-range order, and thus not visible by LEED. As expected from the LEED pattern, the most abundant structure is the $d 6 r$. Close-ups of $1.5 \mathrm{~nm} \times 1.5 \mathrm{~nm}$ sections of the large scale image showing double $N$-membered rings are shown in insets $\mathbf{a}(d 6 r), \mathbf{b}$ $(d 4 r$ and $d 8 r)$ and $\mathbf{c}(d 5 r$ and $d 7 r)$. In these insets, the position of the tetrahedral $\mathrm{T}$ atoms is shown in green while the $\mathrm{O}$ atoms are shown in red. Insets a', b' and c' show a connected-dots representation where the different ring sizes are emphasized by different colors. As it is seen in figure 2, a significant portion of the surface is composed of ordered regions containing only 6 membered rings. These regions were found to account for $\sim 53 \%$ of the surface. The number of rings for each size was counted in a set of images obtained for this aluminosilicate surface with good-enough resolution to clearly distinguish the position of the tetrahedral atoms in most cases. A collage of the images used for counting is included in the supporting information. The size could be clearly identified for 526 rings, out of which 279 were 6-MR located in ordered regions. The ring statistics, excluding the 6-membered rings within the aforementioned ordered regions, are shown in figure 3 as a histogram for ring sizes between 4 and 11. Note that 6-MR (and small clusters of 6-MR) within the regions lacking long-range order are included in the histogram. For comparison, the ring distribution for the vitreous silica film, reported by Lichtenstein et al., [Lichtenstein 2011] is also shown in the same histogram (dashed-line border), normalized to the same number of total rings.

Having established the presence of a slight majority of ordered regions of 6-MR by STM, and that the IRA spectrum indicates that the same structures that are observed by STM are repeated beneath with $\mathrm{O}$ atoms bridging the two layers through $\mathrm{T}-\mathrm{O}-\mathrm{T}$ linkages, we will next proceed to analyze the distribution of rings on the rest of the surface, i.e.: the regions lacking long range order. A striking feature of this system is the fact that in most cases 5-membered rings (5-MR) are located next to 7-MR, while 4-MR are located next to 8-MR (or larger rings). The case of adjacent 5- and 7-MR can be related to Stone-Wales defects which are well-known in hexagonal networks of carbonbased materials, [Stone86] and such motifs have also been shown for the case of a two-dimensional vitreous silica network. [Lichtenstein 2011] The latter system consisted of non-ordered arrangements of double $\mathrm{N}$-membered rings with $\mathrm{N}$ ranging from 4 to 9 , with asymmetric ring size distributions peaking at $d 6 r$ and having $d 5 r$ as the second most abundant species. [Lichtenstein2011] However, for the case reported here, in which aluminum atoms have been incorporated into the framework, there is a significant increase in the population of $d 4 r$ and $d 8 r$, such that the number of instances is comparable to the number of $d 5 r$, $d 7 r$. A silica bilayer film grown on graphene, with vitreous regions similar to the ones reported by Lichtenstein, was reported by Huang et al., in which case an even lower population was found for $d 4 r$. [Huang2012] While $d 5 r$ and $d 7 r$ have been found on pure silica systems, these motifs, although present in many zeolites, do not comprise any of the 23 SBUs. In fact, zeolites show a strong preference for ring sizes having even numbers of tetrahedral atoms, and that is related to Lowenstein's rule, [Lowenstein 54] which states that $\mathrm{Al}-\mathrm{O}-\mathrm{Al}$ moieties are forbidden. This leads, to the formation of $\mathrm{Al}-\mathrm{O}-\mathrm{Si}$ moieties which repeat around the rings, resulting in the observed even-numbered rings. For the limit case of a 1:1 Si:Al ratio, all rings must be even-numbered. This trend showing a preference of evennumbered rings in zeolites was noted already in early works. [Barrer59] Furthermore, the $d 4 r$ and the $d 6 r$ units were then proposed to be present in the "crystallinzing magma" in one of the first references to the synthesis mechanism in 1959 by Barrer et al. [Barrer59] Although STM images allow the determination of the position of tetrahedral atoms, the distinction between $\mathrm{Al}$ and $\mathrm{Si}$ atoms is not clear. Nevertheless, based on the previous discussion, the increase in the number of $d 4 r$ and $d 8 r$ can be clearly attributed to the incorporation of $\mathrm{Al}$ atoms to the framework.

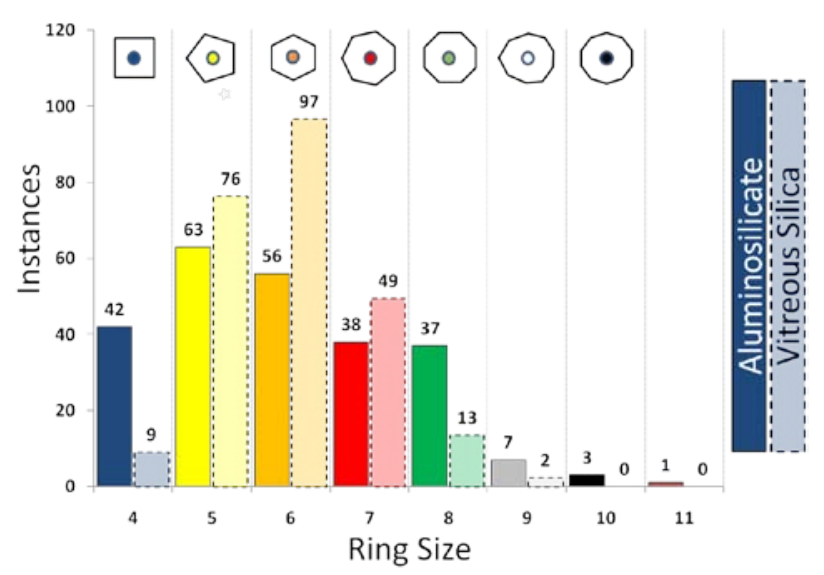

Figure 3. Histogram of ring size distribution for rings in the aluminosilicate film, excluding the 6-MR in the ordered regions. For comparison, the normalized ring distribution for the vitreous silica reported by Lichtenstein et al. is also included (bars with dashedline borders). [Lichtenstein2011] 
In the case of $d 4 r$ units, there is a four-fold increase for the aluminosilicate compared to the vitreous silica. A high population of cubes $(d 4 r)$ has been previously related to the introduction of atoms that allow smaller $\mathrm{T}-\mathrm{O}-\mathrm{T}$ angles into silica networks. [Lobo] For the case pure silica there is a preferential formation of hexagonal networks for the crystalline bilayer structure. For films that do not reach the crystalline state, such as the one reported by Lichtenstein et al., there is also a significant population of 5-MRs and 7-MRs, since these are the ones with the smaller deviation from the tetrahedral angles, after the 6-MR. However, the introduction of atoms, such as Ge, Ga and $\mathrm{Al}$, result in more flexible networks in terms of the $\mathrm{O}-\mathrm{T}-\mathrm{O}$ angles, [Lobo] and this is clearly the case for this system reported here. Although the images do not show different contrasts for the different tetrahedral atoms, it can be safely assumed, based on the previous discussion, that $\mathrm{Al}-\mathrm{O}-\mathrm{Si}-\mathrm{O}-\mathrm{Al}$ linkages are present in the $d 4 r$. The stability of such arrangements was in fact clearly described by the Sauer group, even for low $\mathrm{Al}$ contents. [Schroeder93] They showed, by means of firstprinciples calculations, that for pure silica frameworks the hexagonal prism structure $(d 6 r)$ is more stable than the cube one $(d 4 r)$, [Hill94] while the stability order is reversed for the Alsubstituted case, [Hill95], i.e. the cube structure $(d 4 r)$ is more stable for aluminosilicates.

A motif with alternating $d 4 r$ and $d 8 r$ was often observed when scanning this system (shaded areas with white dashed lines in Figure 2. Figure 4a shows one of the regions with 4 times the alternating $d 4 r$ and $d 8 r$ motif. What follows is a topological exercise with this structure that allows us to relate it to the previously referred transformation between the widely used synthetic zeolite LTA (or zeolite A) and the layered material hexacelsian, with a structure very similar to our film. [Djordjevic2001] A CBU of LTA is the $\alpha$-cage, which is shown in Figure $4 \mathrm{f}$ (note that the $\alpha$-cage is not only found in LTA but it has been found in 7 other framework types). [Atlas2007] A label showing the number of tetrahedral atoms forming the rings is shown in the image in Figure $4 \mathrm{a}$, and a dot-bar representation of the connectivity of the T atoms is shown in Figure $4 \mathrm{~b}$. Since the same structural motif is repeated below for this system, the bottom layer and the connection to the top layer are shown in Figure 4c. Let us now consider a cut-out of a section of this structure (Fig. 4d) and unfold that section such that it becomes now planar as shown in Figure 4e. Strikingly, this planar arrangement of rings, which was contained within our structure, can now be folded into an $\alpha$-cage. This remarkable fact, that the same set of rings with the same connective sequence between rings is shared between this planar framework, and the $\alpha$ cage unit, provides a strong indication about the mechanism by which three-dimensional zeolites can be transformed into planar structures by simple thermal treatments. [Djordjevic2001] It is possible that after the unfolding of the $\alpha$-cage unit into a planar structure upon heating, the resulting alternating sequence of $d 4 r$ and $d 8 r$ could then be converted into the $d 6 r$ units of hexacelsian, following a mechanism analogous to the inverse of the formation of Stone-Wales-like defects. Further studies in this regard could be provided by theory, although this is beyond the scope of this work.

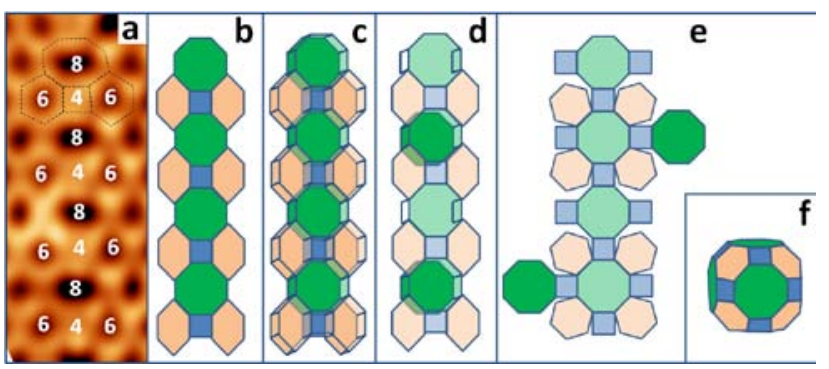

Figure 4. (a) Region with a chain of alternating 4- and 8-membered rings (8-MR). (b) Schematic representation of this region including 6-membered rings adjacent to the chain. 8-MRs are shown in green, 6-MRs in orange and 4-MRs in blue. (c) Three-dimensional representation including the identical layer repeated below. (d) Arbitrary cutout from (c). (e) Unfolded version of the cutout in (d). (f) $\alpha$-cage. This is a CBU of Zeolite A, and can be obtained by folding the structure shown in (e).

The alternating 4-MR and 8-MR structure also serves as a boundary that shifts adjacent crystalline regions by one unit cell of the $\mathrm{Ru}(0001)$ substrate in the direction normal to the chains of 4-MR and 8-MR. Figure 5 shows a clear example of this. There, two crystalline domains can be observed, separated by two different kinds of domain boundaries. One of them, is the previously referred one (4-MR+8-MR) , while the second one consists of alternating pairs 7-MRs and 5-MRs. However, the latter domain boundary runs only $30^{\circ}$ off the direction of the shift. The two crystalline domains are shifted by one $\mathrm{Ru}(0001)$ unit cell. The white arrow indicates the direction of the shift while the white dashed-line is a guide to the eye to ease the visualization of the shift. For comparison, domain boundaries of this kind have not been reported for crystalline silica bilayer systems. For comparison, domain boundaries containing 5-MR and 7-MR have also been observed for crystalline silica bilayer systems (unpublished data). In addition, for the silica bilayer, we have found domains shifted by slightly more than one substrate unit cell, running perpendicular to the direction of the shift, in an analogous manner to the $(4-\mathrm{MR}+8-\mathrm{MR})_{\mathrm{n}}$ domain boundary. However, for the silica case, these domain boundaries did not have 4-MRs but consisted of alternating 8-MRs and pairs of 5-MRs. Note also that these $(8-\mathrm{MR}+2 \times 5-\mathrm{MR})_{\mathrm{n}}$ boundaries run along the main $\mathrm{Ru}(0001)$ directions, while for the aluminosilicate they are $30^{\circ}$ off. It is clear from analyzing both crystalline and vitreous regions of this film that the incorporation of $\mathrm{Al}$ results in changes in the rings-size distribution favoring the presence of even-numbered rings and especially 4-MR.

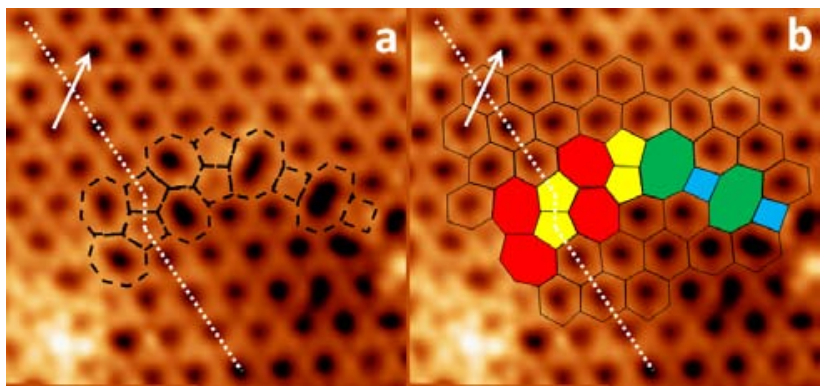

Figure 5. (a) Domain boundary with rings emphasized by dashed line. (b) Boundary rings colored according to size in order to ease 
the visualization of the boundary structure. The white arrow indicates the direction of the shift. The white dashed line is a guide to the eye to visualize the shift.

In summary, a framework of polygonal prisms of tetrahedral atoms, which constitute the building blocks of significant number of zeolites were identified with atomic resolution in a flat aluminosilicate. A substantial increase is observed in the population of prisms with even-numbered rings, when compared to analogous pure silica systems. These units had been previously proposed as precursors for the zeolite formation. In particular, the number of double four-membered rings (cubes) shows a significant increase. In addition, close topological relations between this planar aluminosilicate and Zeolite A were shown, which can lead to explanations of the mechanisms of the transformations between these systems.

\section{ASSOCIATED CONTENT}

Supporting Information. Further experimental details, XPS spectra for $\mathrm{Al}$ and $\mathrm{Si}$, IRA spectrum, collage of STM images with ring assignments, histogram of ring distribution including all regions and STM images describing domain boundaries are included as supporting information. This material is available free of charge via the Internet at http://pubs.acs.org.

\section{AUTHOR INFORMATION}

\section{Corresponding Author}

bosco@fhi-berlin.mpg.de.

\section{Notes}

The authors declare no competing financial interest.

\section{ACKNOWLEDGMENT}

This work has been supported by the German Science Foundation (DFG). We gratefully acknowledge Prof. J. Sauer and L. Lichtenstein for useful discussions. J.A. Boscoboinik gratefully acknowledges a fellowship by the Alexander von Humboldt Foundation.

\section{ABBREVIATIONS}

SBU, secondary building unit; CBU, composite building unit; MR, membered ring; LEED, low energy electron diffraction; XPS, x-ray photoelectron spectroscopy; STM, scanning tunneling microscopy; IRAS, infrared reflection-absorption spectroscopy; DFT, density functional theory; $d x r$, double $x$-membered ring.

\section{REFERENCES}

[Smith88] Smith, J. Chem. Rev. 1988, 88, 149.

[Cejka2010] Zeolites and catalysis. Editors: Jiri Cejka, Stacey Zones, Avelino Corma. John Wiley \& Sons, 2010.

[Baerlocher] Ch. Baerlocher and L.B. McCusker, Database of Zeolite Structures: http://www.iza-structure.org/databases/

[Foster] M.D. Foster, M.M.J. Treacy A Database of Hypothetical Zeolite Structures: http://www.hypotheticalzeolites.net.

[Löffler10] D. Löffler, J. Uhlrich, M. Baron, B. Yang, X. Yu, L. Lichtenstein, L. Heinke, C. Büchner, M. Heyde, S. Shaikhutdinov, H.-J. Freund, R. Włodarczyk, M. Sierka, J. Sauer Phys. Rev. Lett. 2010, 105, 146104.

[Lichtenstein2011] L. Lichtenstein, C. Buchner, B. Yang, S. Shaikhutdinov, M. Heyde, M. Sierka, R. Włodarczyk, J. Sauer, H.-J. Freund Angew. Chem. Int. Ed. 2011, 50, 1-5.
[Włodarczyk 2012] R. Włodarczyk, M. Sierka, J. Sauer, D. Löffler, J. J. Uhlrich, X. Yu, B. Yang, I. M. N. Groot, S. Shaikhutdinov, H. J. Freund, Phys. Rev. B 2012, 85, 085403.

[Lowenstein54] W. Lowenstein, Am. Mineral. 1954, 39, 92.

[Atlas2007] Ch. Baerlocher, L.B. McCusker and D.H. Olson Atlas of Zeolite Framework Types, $6^{\text {th }}$ revised edition, 2007. http://www.izastructure.org/databases/books/Atlas_6ed.pdf

[Frank01] M. Frank, K. Wolter, N. Magg, M. Heemeier, R. Kühnemuth, M. Bäumer, H.-J. Freund, Surf. Sci. 492 (2001) 270.

[Rotole98] John A. Rotole and Peter M. A. Sherwood. Surface Science Spectra, Vol. 5, 1998.

[Grünert94] J . Phys. Chem. 1994, 98, 10920-10929.

[Schroeder93] Schroeder, K.P.; Sauer, J. J. Phys. Chem. 1993, 97 (25), 6579.

[Yoshiki51] Yoshiki, B.; Matsumoto, K. J. Am. Ceram. Soc. 1951, $34,283$.

[Hill94] Joerg R. Hill, Joachim Sauer, J. Phys. Chem. 1994, 98, $1238-1244$

[Hill95] Joerg-R. Hill, Joachim Sauer, J. Phys. Chem. 1995, 99, 9536-9550.

[Bosco2012] J.A. Boscoboinik, X. Yu, B. Yang, F.D. Fischer, R. Włodarczyk, M. Sierka, S. Shaikhutdinov, J. Sauer, H.-J. Freund Angew. Chem. Int. Ed. 2012, In Press.

[Wagner81] Wagner, C.D.; Davis, L.E.; Zeller, M.V.; Taylor, J.A.; Raymond, R.H.; Gale, L.H. Surface and Interface Analysis 1981, 3, 211

[Huang2012] Pinshane Y. Huang, Simon Kurasch, Anchal Srivastava, Viera Skakalova, Jani Kotakoski, Arkady V. Krasheninnikov, Robert Hovden, Qingyun Mao, Jannik C. Meyer, Jurgen Smet, David A. Muller, Ute Kaiser, Nano Lett., 2012, 12, 1081-1086.

[Lowenstein54] W. Lowenstein, Am. Mineral. 1954, 39, 92.

[Lobo] R.F. Lobo Chapter 3 of Handbook of Zeolite Science and Tech nology. Eds.: S.M. Auerbach, K.A. Carrado, P.K. Dutta. Marcel Dekker, Inc. 2003.

[Djordjevic2001] J. Djordjevic, V. Dondur, R. Dimitrijevic, A. Kremenovic Phys. Chem. Chem. Phys., 2001, 3, 1560-1565

[Eichler97] U. Eichler, M. Brändle, J. Sauer, J. Phys. Chem. B 1997, $101,10035-10050$.

[Brändle98] M. Brändle, J. Sauer, J. Am. Chem. Soc. 1998, 120, 15561570.

[Katada2009] N. Katada, K. Suzuki, T. Noda, G. Sastre, M. Niwa, J. Phys. Chem. C 2009, 113, 19208-19217.

[Cundy2005] C.S. Cundy, P.A. Cox Micropor. Mesopor. Mat. 2005, $82,1-78$.

[Barrer59] R.M. Barrer, J.W. Baynham, F.W. Bultitude, W.M. Meier J. Chem. Soc. 1959, 195.

[Stone86] Stone, A. J.; Wales, D. J. Chem. Phys. Lett. 1986, 128, 501. 
Table of Contents (TOC)

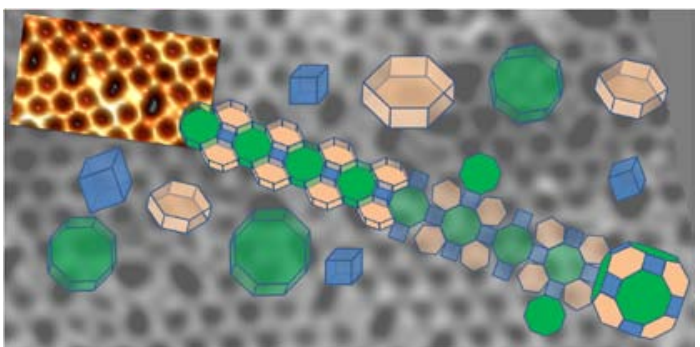

